\title{
Long Cut Straw Provides Stable the Rates of Survival, Pregnancy and Live Birth for Vitrification of Human Blasotcysts
}

\author{
Jung-Woo Lee ${ }^{1,2},{ }^{\dagger}$ Jeong-Ho Cha ${ }^{1}$, Sun-Hee Shin ${ }^{1}$, Yun-Jeong Kim ${ }^{1}$, Seul-Ki Lee ${ }^{1}$, Hye-Jin Cha ${ }^{1}$, \\ Ji-Hae Kim ${ }^{1}$, Ji-Hyun Ahn' ${ }^{1}$, Hye-Young Kim ${ }^{1}$, Kyung-Ah Pak ${ }^{1}$ Ji-Sung Yoon ${ }^{1}$, \\ Seo-Young Park ${ }^{1}$ and ${ }^{\dagger}$ Choon-keun Park ${ }^{2}$ \\ ${ }^{I}$ Agaon Fertility Clinic, Seoul 08391, Korea \\ ${ }^{2}$ College of Animal Life Science, Kangwon National University, Chuncheon 24341, Korea
}

\begin{abstract}
Most of the commercial devices for vitrification are directly immersed into the warming solution (WS) for increasing of warming rate. However, the previous modified cut standard straw (MCS) which has reported is difficult to immerse into the WS. The aim of this study was to investigate whether the long cut straw (LCS) could be useful as a stable tool for vitrified-warmed human blastocysts. A total of 138 vitrified-warmed cycles were performed between November 2013 and November 2014 (exclusion criteria: women $\geq 38$ years old, poor responder, surgical retrieval sperm, and severe male factor). The artificial shrinkage was conducted using 29 -gauge needles. Ethylene glycol and dimethyl sulfoxide (7.5\% and $15 \%$ $(\mathrm{v} / \mathrm{v}))$ were used as cryoprotectants. Freezing and warming were conducted using the LCS tool. The cap of LCS was removed using the forceps in the liquid nitrogen $\left(\mathrm{LN}_{2}\right)$ and then directly immersed into the first WS for 1 min at $37^{\circ} \mathrm{C}(1 \mathrm{M}$ sucrose). Only re-expanded blastocysts were transferred after it was cultured in sequential media for 18-20 h. A total of 294 blastocysts were warmed, and all were recovered (100\%). Two hundred eighty-five embryos were survived (96.9\%). The vitrifiedwarmed blastocysts of all patients were transferred without any cancellation. We were able to achieve a reasonable implantation (24.2\%), following by clinical pregnancy (36.2\%), which then continued to ongoing pregnancy (36.2\%), and live birth (31.2\%). Using LCS is achieved the acceptable rates of survival, pregnancy and live birth. Therefore, the LCS could be considered as a stable and simple tool for human embryo vitrificaton.
\end{abstract}

Key words : Human blastocysts, Long cut straw, Vitrification, Survival, Clinical outcomes

\section{INTRODUCTION}

As a one of cryopreservation methods, vitrification that does not require any expensive equipment and long working hours is globally used for preservation of human embryos in fertility clinic (Loutradi et al., 2008; Youssry et al.,
2008). This method can prevent potential damages in embryos by reducing ice crystallization (Rall \& Fahy, 1985). The successful vitrification is influenced by various factors including the types of tools, conditions of freezing and thawing process, cryoprotectants, exposure time in equilibration solutions and storage methods (Vajta \& Nagy, 2006).

\footnotetext{
Manuscript received July 18, 2016, Received in revised form August 12, 2016, Accepted September 19, 2016

${ }^{\dagger}$ Corresponding Author : Jeong-Ho Cha, Agaon Fertility Clinic, Seoul 08391, Korea. Tel. : +82-2-864-7500, Fax : +82-2-864-7550, E-mail : ivfcha@naver.com Choon-Keun Park, College of Animal Life Science, Kangwon National University, Chuncheon 24341, Korea. Tel. : +82-33-250-8627, Fax : +82-33-2505574, E-mail : parkck@kangwon.ac.kr

This is an Open Access article distributed under the terms of the Creative Commons Attribution Non-Commercial License (http:// creativecommons.org/licenses/by-nc/3.0) which permits unrestricted non-commercial use, distribution, and reproduction in any medium, provided the original work is properly cited.
} 
In the past few years, many researchers have been studied about cryopreservation tools that are divide open-type and closed-type as a one of important factor. During the freezing process using open tool, embryos directly contact to liquid nitrogen $\left(\mathrm{LN}_{2}\right)$ and this contact cause high freezing rate and reduction of chilling injury (Vajta \& Kuwayama, 2006). However, potential risk of cross-contamination by direct contact between embryo and $\mathrm{LN}_{2}$ during cryopreservation had been reported (Bielanski et al., 2000, Vanderzwalmen et al., 2010). On the contrary, closed tools could prevent infection agents and disease transfer because it eliminates direct contact between the embryos and $\mathrm{LN}_{2}$. However, closed tools cause reduction of low freezing rate (Vajta et al., 2015, De Munck et al., 2016). Although there are still questionable and controversial about between open and closed tools, many oocytes and embryos in human are usually vitrified with open tools worldwide (Vajta et al., 2015).

The modified cut standard straw (MCS) is one of handmade open tools. A few studies have recently been reported that MCS is used for vitrification of mouse oocytes, embryos and human embryos (Kim et al., 2010; Shin et al., 2011; Lim et al., 2013; Jung \& Cheon, 2014). As a one of commercial tools for vitrification, MCS was directly immersed into the warming solution (WS) for increasing of warming rate (Vajta et al., 2015) and it easy to use. However, the existing MCS is difficult to immerse into the WS (Fig. 3), thereby embryologists grab the MCS for warming before dipping into WS.

Therefore, we modified the structure of the MCS to solve the inconvenience and this modified straw was called the 'Long Cut Straw (LCS)'. This study was conducted to investigate whether the LCS could be useful as a stable tool for vitrified-warmed human blastocysts.

\section{MATERIALS AND METHODS}

\section{Participants}

A total of 138 vitrified-warmed cycles were used for investigation between November 2013 and November 2014 in this study. Women, who are $\geq 38$ years old of age, are either poor responder and with husbands who have severe male factor and surgical retrieval sperm were excluded. This study was approved by the Agaon Fertility Clinic Institutional Review Board (IRB reference number Agaon IRB-15-003).

\section{Ovarian stimulation and oocytes collection}

Ovarian stimulation was conducted with either a gonadotropin releasing hormone $(\mathrm{GnRH})$ agonist long protocol or a GnRH antagonist (Merck Serono, Italy) protocol. Human chorionic gonadotropin (hCG, 10,000 IU, Merck Serono, Italy) was injected to the women 36 hours before ovum pick-up. Oocytes were collected via transvaginal ultrasoundguided with a 19-gauge needle (Dukwoo Medical, Korea).

\section{Insemination oocytes and culture of zygotes}

Mature oocytes were inseminated with either conventional insemination or intracytoplasmic sperm injection (ICSI), depending on the state of sperm, oocyte and previous history of patient, etc. The outcome of fertilization was assessed in 17-19 hours after insemination, when the presence of two pronuclei was observed. Up to three zygotes were cultured in $30 \mu \mathrm{L}$ of micro-drop with either one-step medium (Life Global $^{\circledR}$, Belgium) or sequential medium (Vitrolife, Sweden) under covering with paraffin oil (Vitrolife, Sweden) in the atmosphere of $6 \% \mathrm{CO}_{2}, 5 \% \mathrm{O}_{2}$ and $95 \%$ humidity at $37^{\circ} \mathrm{C}$.

\section{The making process of the LCS}

The LCS is pulled at the end part of a $0.25 \mathrm{~mL}$ straw (total length: $13 \mathrm{~cm}$, cutting part: $1.5 \mathrm{~cm}$ ) by heating using an alcohol lamp and then cutting it gentle at an angle (Fig. 3). The end part of the sealed cap was marked blue to distinguish the part in the $\mathrm{LN}_{2}$ (Fig. 1). The LCS was sterilized using ethylene oxide and then used it after two weeks. 


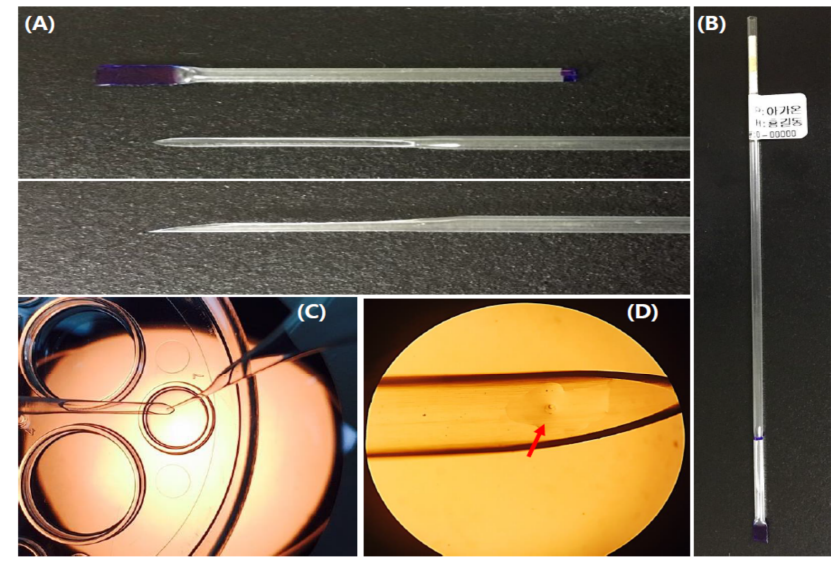

Fig. 1. Vitrification-warming of blastocysts using the long cut straw (LCS) and structure. (A) Shape of the cap and cutting part. (B) Shape of the LCS. (C) Loading method. (D) Loaded blastocysts on the cutting part of the LCS with a minimum volume (×40). Red allow: Blastocyst.

\section{Vitrification and artificial shrinkage procedure}

Surplus embryos were cultured, until day 6 , to the blastocyst stage after embryo transfer (ET). The blastocysts graded 36 were cryopreserved by Gardner and Schoolcraft's blastocyst scoring system (Gardner et al., 2000). Ethylene glycol (EG, Sigma-Aldrich, USA) and dimethyl sulfoxide (DMSO, SigmaAldrich, USA) were used as the vitrification solution (VS) which acts as the permeable cryoprotectants. The artificial shrinkage was performed using 29-gauge needles before vitrification of balstocysts. For the vitrification procedure, one to two blastocysts were placed into the VS \#1 (PBS (SAGE, USA) supplemented with 7.5\% EG + 7.5\% DMSO + $20 \%$ SSS (IrvineScientific, USA)) for $1 \mathrm{~min}$ at room temperature (RT). The blastocyst was then transferred into the VS \#2 (PBS supplemented with 15\% EG + 15\% DMSO + $20 \%$ SSS $+0.5 \mathrm{M}$ Sucrose) for 25-30 second at RT. The blastocyst was then loaded onto the end part of the LCS as described (Cryotop method) by M. Kuwayama et al. (2005b). The blastocyst around VS \#2 was nearly removed using pipette to make minimum volume size (Fig. 4) and then the LCS was plunged into $\mathrm{LN}_{2}$ after putting a cap on.

\section{Warming of vitrified balstocyst}

For warming, the sealed cap of the LCS was removed using the forceps inside the $\mathrm{LN}_{2}$ and then directly immersed into the first $1 \mathrm{M}$ sucrose solution for $1 \mathrm{~min}$ at $37^{\circ} \mathrm{C}$. The blastocyst was transferred by sequential exposure into the second $0.5 \mathrm{M}$ sucrose solution for $3 \mathrm{~min}$ and was transferred into $0.25 \mathrm{M}$ sucrose solution for $2 \mathrm{~min}$, and lastly into the washing medium (PBS supplemented with 20\% SSS) for 5 min at RT. The survival of vitrified-warmed blastocyst (VWB) was assessed under a stereoscopic microscope (Olympus, Japan) around 18-20 hours after warming.

\section{Embryo transfer and pregnancy}

Only re-expanded blastocyst transfer was performed using an ET catheter $\left(\mathrm{COOK}^{\circledR}\right.$ Medical, Australia). Endometrium was prepared for VWB transfer either by artificial cycle protocols with exogenous estrogens or by natural cycles. In artificial cycle protocols, progesterone in oil $(50 \mathrm{mg} /$ day $)$ was administered on daily basis when the endometrial thickness reached $8 \mathrm{~mm}$ or more, and the thawed embryos were transferred 5 days later. In natural cycle, the endocrine preparation of the endometrium is achieved by endogenous estrogen production from a developing follicle. Timing of embryo transfer is determined by administering $\mathrm{hCG}$ for triggering ovulation. One or two re-expanded blastocysts were transferred into uterus after 6 days of progesterone treatment. The rates of implantation and clinical pregnancy were confirmed by monitoring of the gestational sac at 6 weeks of pregnancy. Ongoing pregnancy was defined as showing a positive heart-beat at ultrasound scan after 7 weeks of gestation. Live birth outcomes including number of babies were investigated.

\section{RESULTS}

A total of 138 vitrified-warmed cycles had altogether 294 blastocysts vitrified using the LCS method. The mean 
J-W Lee, J-H Cha, S-H Shin, Y-J Kim, S-K Lee, H-J Cha, J-H Kim, J-H Ahn, H-Y Kim, K-A Pak, J-S Yoon, S-Y Park, C-k Park

Table 1. Clinical outcomes of vitrified-warmed blastocyst using long cut straw (LCS)

\begin{tabular}{lc}
\hline \multicolumn{1}{c}{ Parameter } & Value \\
\hline No. of cycles & 138 \\
No. of patients & 112 \\
Average patients age $(\mathrm{y} \pm \mathrm{SD})$ & $32.3 \pm 2.5$ \\
No. of embryos thawed & 294 \\
No. of embryos recovered & $294(100 \%)$ \\
No. of embryos survived & $285(96.9 \%)$ \\
No. of cycles canceled & 0 \\
Average no. of embryos transferred & $2.1 \pm 0.4$ \\
$\quad$ per cycle & \\
Implantation rate & $69 / 285(24.2 \%)$ \\
Clinical pregnancy rate & $50 / 138(36.2 \%)$ \\
Ongoing pregnancy rate & $50 / 138(36.2 \%)$ \\
Live birth rate & $43 / 138(31.2 \%)$ \\
\hline
\end{tabular}

age was $32.3 \pm 2.5$. A total of 294 blastocysts that were warmed, 294 blastocysts were recovered (100\%), and 285 of those blastocyst were survived (96.9\%).

ET was performed in all cases without cancellation. The mean number of transferred embryos was $2.1 \pm 0.4$. Mean implantation rate was $24.2 \%$. The rate of clinical pregnancy was $36.2 \%$, and $50(36.2 \%)$ of those patients had an ongoing pregnancy with fetal heartbeat at 12 weeks of gestation. The rate of live birth was $31.2 \%$. These results are presented in Table 1.

\section{DISCUSSION}

We described the simple warming method of the LCS as a useful and stable tool. LCS was directly immersed into the WS without pre-warming by performer's hands and use of this tool showed the high recovery and survival rates for the VWB, followed by successful pregnancy and live birth. This study is first report regarding live birth after using the modified straw.

Kim et al. (2010) and Lim et al. (2013) reported that the survival rates of VWB using the MCS were $73.0 \%$ and $75.6 \%$, respectively. On the other hand, our data showed higher survival rate of VWB (96.9\%) using LCS than vitrified-warmed using MCS in other study. In addition, the results of present study showed a reasonable ongoing pregnancy $(36.2 \%)$ and live birth $(31.2 \%)$ rate of transferred VWB, which was vitrified-warmed by LCS, in human.

The end part of MCS have a narrow and short surface that caused occurrence of capillary action (Fig. 3C). Because of this structure, it is difficult to immerse into the WS. Therefore, the part of straw cap is removed using hands to grab it at performer's hand temperature (Fig. 2B) Therefore, the end part of vitrified MCS is warmed using performer's hand and then VWB are transferred using the pipette into the WS. During the pre-warming process, VWB are exposed to high concentration of cryoprotectant solution and it caused the risk of lost warmed-embryos. The chemical toxicity of cryoprotectant increased the potential risk of injury in vitrified human blastocyst (Mukaida et al., 2006). However, the LCS can solve these problems by elimination of unnecessary process of additional exposure to cryoprotectant.

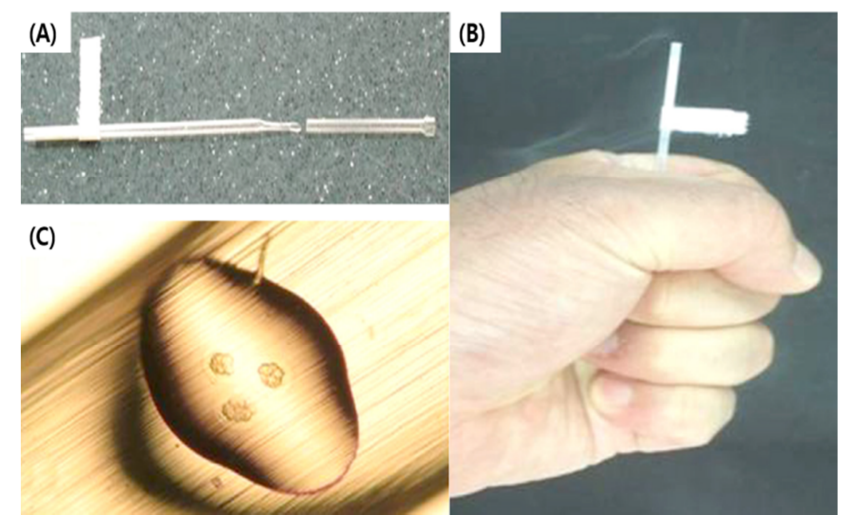

Fig. 2. Vitrification-warming of embryos using the modified cut standard straw (MCS) and structure. (A) Shape of the MCS. (B) Warming of embryos in the MCS by holding the MCS with bare hands. (C) Loaded embryos on the tip of the MCS with a minimum volume of vitrification solution $(\times 40)$. (Lim et al., 2013). 
Previous study reported that the shape of in-house-made tools were difficult to make small volume of cryoprotectant for vitrification (Marco-Jiménez et al., 2016). However, the LCS is possible to load the minimum volume size $(<0.1$ $\mu \mathrm{L}$ ) of cryoprotectant with blastocyst on the surface (Fig. 4B) because the end part of LCS with long length has a similar structure to the Cryotop as described by M. Kuwayama et al. (2005b). A lower volume of cryoprotectant during vitrification can reduced the rates of ice crystallization and chilling injury by increase of freezing rates (Cobo et al., 2008). Therefore, we expected that using the LCS might raise the speed of freezing and warming as commercial tools.

Some researchers have voiced out their concerns about

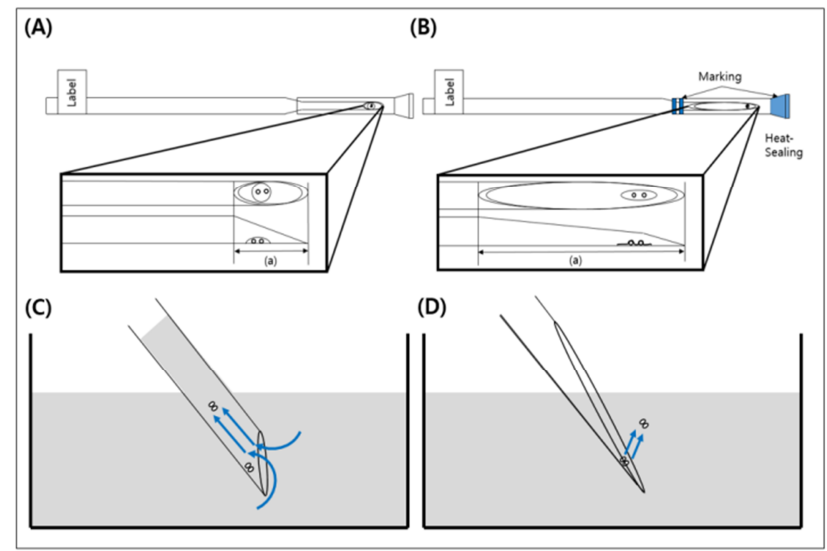

Fig. 3. Comparison of the modified cut standard straw (MCS) and the Long Cut Straw (LCS) after warming. Schematic structure of the MCS (A) and the LCS (B). (C) Image of suction warmed-blastocysts after warming into the MCS as capillary action. (D) Image of warmed-blastocysts after warming using the LCS, (a) Embryos are loaded onto the straw.
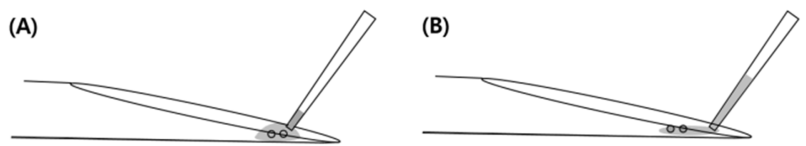

Fig. 4. Schematic diagram of loading process using the Long Cut Straw (LCS). (A) Load the blastocyst on the distal end of the LCS. (B) Aspirate the blastocyst around VS \#2 $(<0.1 \mu \mathrm{L})$ using pipette. the safety of the open tools for the freezing of sperm or embryo because there is a potential risk of cross-contamination (Bielanski et al., 2000; Bielanski et al., 2003; Bielanski \& Vajta, 2009; Kuwayama et al., 2005a; Vanderzwalmen et al., 2010). As the LCS is an open tool, we cannot eliminate the potential risk of cross-contamination completely. However, the recent guideline of the American Society for Reproductive Medicine (2013) has stated this: "there also are theoretic infectious disease concerns with the use of open vitrification methods. However, infectious transmission has never been observed in reproductive tissues from this technique". We thought that the guideline supports to resolve about a concern by the use of open tools. In addition, during the past few years, tens thousands of women had delivered healthy babies worldwide using the open tools (Vajta et al., 2015). Despite using the open tools for vitrification is a still questionable and controversial, this tools are still used to freeze oocytes and embryos worldwide.

In our center, the frozen embryos and sperm derived from any infected patients were separately stored from the frozen embryo and sperm derived from all other non-infected patients, using different containers. This is necessary to minimize any potential risk of cross-contamination.

The cost of the LCS is much lower than that of commercial kits (Gvakharia \& Adamson, 2011). We expected that use of LCS, as a cost factor of cryopreservation, would lighten the financial burden of patients. In addition, the LCS is user-friendly and easy to use. We suggest that the LCS tool, which were used for vitrification of blastocyst stage, could provide us with acceptable clinical outcomes, live birth and has simplified the warming process. Further investigations on comparative studies regarding clinical outcome among various tools and larger data are needed.

\section{ACKNOWLEDGEMENT}

We thank the anonymous referees for their useful suggestions. 
J-W Lee, J-H Cha, S-H Shin, Y-J Kim, S-K Lee, H-J Cha, J-H Kim, J-H Ahn, H-Y Kim, K-A Pak, J-S Yoon, S-Y Park, C-k Park

\section{REFERENCES}

Bielanski A, Bergeron H, Lau P, Devenish J (2003) Microbial contamination of embryos and semen during long term banking in liquid nitrogen. Cryobiology 46:146-152.

Bielanski A, Nadin-Davis S, Sapp T, Lutze-Wallace C (2000) Viral contamination of embryos cryopreserved in liquid nitrogen. Cryobiology 40:110-116.

Bielanski A, Vajta G (2009) Risk of contamination of germplasm during cryopreservation and cryobanking in IVF units. Hum Reprod 24:2457-2467.

Cobo A, Kuwayama M, Pérez S, Ruiz A, Pellicer A, Remohí J (2008) Comparison of concomitant outcome achieved with fresh and cryopreserved donor oocytes vitrified by the Cryotop method. Fertil Steril 89:1657-1664.

De Munck N, Santos-Ribeiro S, Stoop D, Van de Velde H, Verheyen G (2016) Open versus closed oocyte vitrification in an oocyte donation programme: A prospective randomized sibling oocyte study. Hum Reprod 31:377-384.

Gardner DK, Lane M, Stevens J, Schlenker T, Schoolcraft WB (2000) Blastocyst score affects implantation and pregnancy outcome: towards a single blastocyst transfer. Fertil Steril 73:1155-1158.

Gvakharia M, Adamson GD (2011) Implementation of an inexpensive method of vitrification and warming of human cleavage-stage embryos using cut standard straws. Fertil Steril 95:2552-2553.

Jung YJ, Cheon YP (2014) Improvement of the vitrification method suppressing the disturbance of meiotic spindle and chromosome systems in mature oocytes. Dev Reprod 18:117-125.

Kim HJ, Kim CH, Lee JY, Kwon JH, Hwang D, Kim KC (2010) Effect of cryopreservation day on pregnancy outcomes in frozen-thawed blastocyst transfer. Korean J Reprod Med 37:57-64.

Kuwayama M, Vajta G, Ieda S, Kato O (2005a) Comparison of open and closed methods for vitrification of human embryos and the elimination of potential contamination. Reprod Biomed Online 11:608-614.

Kuwayama M, Vajta G, Kato O, Leibo SP (2005b) Highly efficient vitrification method for cryopreservation of human oocytes. Reprod Biomed Online 11:300-308.

Lim JG, Heo YT, Min SG, Min BY, Uhm SJ, Kim NH (2013) Clinical outcomes of vitrified-thawed embryo transfer using a pull and cut straw method. Obstet Gynecol Sci 56:182-189.

Loutradi KE, Kolibianakis EM, Venetis CA, Papanikolaou EG, Pados G, Bontis I, Tarlatzis BC (2008) Cryopreservation of human embryos by vitrification or slow freezing: A systematic review and meta-analysis. Fertil Steril 90:186-193.

Marco-Jiménez F, Jiménez-Trigos E, Almela-Miralles V, Vicente JS (2016) Development of cheaper embryo vitrifycation device using the minimum volume method. PLOS one 11:1-9.

Mukaida T, Oka C, Goto T, Takahashi K (2006) Artificial shrinkage of blastocoeles using either a micro-needle or a laser pulse prior to the cooling steps of vitrification improves survival rate and pregnancy outcome of vitrified human blastocysts. Hum Reprod 21:3246-3252.

Rall WF, Fahy GM (1985) Ice-free cryopreservation of mouse embryos at $-196^{\circ} \mathrm{C}$ by vitrification. Nature $313: 573-575$.

Shin MR, Choi HW, Kim MK, Lee SH, Lee H, Lim CK (2011) In vitro development and gene expression of frozen-thawed 8-cell stage mouse embryos following slow freezing or vitrification. Clin Exp Reprod Med 38: 203-209.

Vajta G, Kuwayama M (2006) Improving cryopreservation systems. Theriogenology 65:236-244.

Vajta G, Nagy ZP (2006) Are programmable freezers still needed in the embryo laboratory? Review on vitrification. Reprod Biomed Online 12:779-796.

Vajta G, Rienzi L, Ubaldi FM (2015) Open versus closed systems for vitrification of human oocytes and embryos. 
Reprod Biomed Online 30:325-333.

Vanderzwalmen P, Zech N, Prapas Y, Panagiotidis Y, Papatheodorou A, Lejeune B, Jareno D, Vanderzwalmen S, Ectors F (2010) Closed carrier device: A reality to vitrify oocytes and embryos in aseptic conditions. Gynecol
Obstet Fertil 38:541-546.

Youssry M, Ozmen B, Zohni K, Diedrich K, Al-Hasani S (2008) Current aspects of blastocyst cryopreservation. Reprod Biomed Online 16:311-320. 